\title{
On the quotient moment of lower generalized order statistics and characterization
}

\author{
D. KUMAR
}

\begin{abstract}
In this paper we consider general class of distribution. Recurrence relations satisfied by the quotient moments and conditional quotient moments of lower generalized order statistics for a general class of distribution are derived. Further the results are deduced for quotient moments of order statistics and lower records and characterization of this distribution by considering the recurrence relation of conditional expectation for general class of distribution satisfied by the quotient moment of the lower generalized order statistics.
\end{abstract}

Mathematics Subject Classification 2010: 62G30, 62E10

Additional Key Words and Phrases: Lower generalized order statistics, order statistics, record values, general class of distribution, recurrence relations and characterization.

\section{INTRODUCTION}

Kamps [15] introduced the concept of generalized order statistics (gos). It is known that ordinary order statistics, sequential order statistics, Stigler's order statistics and upper record values are special cases of gos. In this article we will consider the lower generalized order statistics ( $($ gos $)$ defined as follows:

Let $n \in N, k \geq 1, m \in \mathfrak{R}$, be the parameters such that

$$
\gamma_{r}=k+(n-r)(m+1)>0 \text { for all } 1 \leq r \leq n
$$

Then $X^{*}(1, n, m, k), \ldots, X^{*}(n, n, m, k)$ are called $l g o s$ from an absolutely continuous distribution function $(d f) F(x)$ with the probability distribution function (pdf) $f(x)$ if their joint $p d f$ has the form

$k\left(\prod_{j=1}^{n-1} \gamma_{j}\right)\left(\prod_{i=1}^{n-1}\left[F\left(x_{i}\right)\right]^{m} f\left(x_{i}\right)\right)\left[F\left(x_{n}\right)\right]^{k-1} f\left(x_{n}\right)$

for $F^{-1}(1)>x_{1} \geq x_{2} \geq \ldots \geq x_{n}>F^{-1}(0)$. 
The marginal $p d f$ of $r-$ th $l g o s, X^{*}(r, n, m, k)$, is

$f_{X^{*}(r, n, m, k)}(x)=\frac{C_{r-1}}{(r-1) !}[F(x)]^{\gamma_{r}-1} f(x) g_{m}^{r-1}(F(x))$.

The joint $p d f$ of $X^{*}(r, n, m, k)$ and $X^{*}(s, n, m, k), 1 \leq r<s \leq n$, is expressed from (1.1) as

$$
\begin{gathered}
f_{X^{*}(r, n, m, k), X^{*}(s, n, m, k)}(x, y)=\frac{C_{s-1}}{(r-1) !(s-r-1) !}[F(x)]^{m} f(x) g_{m}^{r-1}(F(x)) \\
\times\left[h_{m}(F(y))-h_{m}(F(x))\right]^{s-r-1}[F(y)]^{\gamma_{s}-1} f(y), \quad x>y,
\end{gathered}
$$

Let $X^{*}(r, n, m, k), r=1,2, \ldots, n$ be $l$ gos from a continuous population with $d f$ $F(x)$ and $p d f f(x)$, then the conditional $p d f$ of $X^{*}(s, n, m, k)$ given $X^{*}(r, n, m, k)=x, 1 \leq r<s \leq n$, in view of (1.2) and (1.3), is

$$
\begin{aligned}
f_{X^{*}(s, n, m, k) \mid X^{*}(r, n, m, k)}(y \mid x) & =\frac{C_{s-1}}{(s-r-1) ! C_{r-1}}\left[\left(h_{m}(F(y))-h_{m}(F(x))\right]^{s-r-1}\right. \\
& \times \frac{[F(y)]^{\gamma_{s}-1}}{[F(x)]^{\gamma_{r}+1}} f(y), \quad x>y
\end{aligned}
$$

and the conditional $p d f$ of $X^{*}(r, n, m, k)$ given $X^{*}(s, n, m, k)=y, 1 \leq r<s \leq n$, is

$$
\begin{aligned}
& f_{X^{*}(r, n, m, k) \mid X^{*}(s, n, m, k)}(x \mid y)=\frac{(s-1) !}{(r-1) !(s-r-1) !} \frac{g_{m}^{r-1}(F(x))}{g_{m}^{s-1}(F(y))} f(x) \\
& \quad \times[F(x)]^{m}\left[\left(h_{m}(F(y))-h_{m}(F(x))\right]^{s-r-1}\right.
\end{aligned}
$$

where

$$
\begin{aligned}
& C_{r-1}=\prod_{i=1}^{r} \gamma_{i}, \gamma_{i}=k+(n-i)(m+1) \\
& h_{m}(x)=\left\{\begin{array}{lc}
-\frac{1}{m+1} x^{m+1}, & m \neq-1 \\
-\ln x, & m=-1
\end{array}\right.
\end{aligned}
$$


and

$$
g_{m}(x)=h_{m}(x)-h_{m}(1), x \in[0,1) .
$$

We shall also take $X^{*}(0, n, m, k)=0$. If $m=0, k=1$, then $X^{*}(r, n, m, k)$ reduces to the $(n-r+1)$-th order statistic, $X_{n-r+1: n}$ from the sample $X_{1}, X_{2}, \ldots, X_{n}$ and when $m=-1$, then $X^{*}(r, n, m, k)$ reduces to the $r-$ th lower $k$ record value (Pawlas and Szynal, [26]). The work of Burkschat et al. [5] may also refer for $l$ gos. Recurrence relations are interesting in their own right. They are useful in reducing the number of operations necessary to obtain a general form for the function under consideration. Furthermore, they are used in characterizing distributions, which in important area, permitting the identification of population distribution from the properties of the sample.

Recurrence relations for single and product moment of lower generalized order statistics from the inverse Weibull distribution are derived by Pawlas and Szynal [26]. Khan and Kumar [17,18 \& 19], Kumar [20, 21] have established recurrence relations for moments of lower generalized order statistics from exponentiated Pareto, gamma, generalized exponential, Kumaraswamy and J-shaped distributions. Ahsanullah [2] and Mbah and Ahsanullah [25] characterized the uniform and power function distributions based on distributional properties of lower generalized order statistics respectively. El-Din and Kotb [8] have established recurrence relations for quotient moments of generalized order statistics and characterization. Lee and Chang [22, $23 \& 24]$ and Chang [6] have derived recurrence relations of quotient moments of exponential distribution, Pareto distribution, power function distribution and Weibull distribution by record value respectively. Characterizations of particular distributions based on the moments and conditional moments of order statistics were presented by some authors such as Wu and Ouyang [27], Grudzien and Szynal [10], Khan and Abouammoh [12], Ahmad [1], Asadi et al. [3], Govindarajulu [9], amongs others. Kamps [13] investigated the importance of recurrence relations of order statistics in characterization. Characterizations based on gos have been studied by some authors. Keseling [14] characterized some continuous distributions based on 
conditional distributions of gos. Bieniek and Szynal [4] characterized some distributions via linearity of regression of gos. Cramer et al. [7] gave a unifying approach on characterization via linear regression of ordered random variables. Khan et al. [16] characterized some continuous distributions through conditional expectation of functions of gos.

The aim of the present study is to give some recurrence relations for quotient moments of $l$ gos from general class of distribution. In section 2 we give recurrence relations for quotient moments of general class of distribution. Then we show that results for order statistics and record values are deduced as special cases. In section 3 we give recurrence relations for conditional quotient moments of general class of distribution and we show that results for order statistics and record values are deduced. In last section of the paper we prove a characterization result on this distribution based on recurrence relation for conditional quotient moment of the lower generalized order statistics.

Let the general form of distribution be

$$
F(x)=[a h(x)+b]^{c}, \alpha \leq x \leq \beta
$$

Where $a, b$ and $c$ are such that $F(\alpha)=0, F(\beta)=1$ and $h(x)$ is a monotonic and differentiable function of $x$ in the interval $[\alpha, \beta]$.

Then

$$
f(x)=\frac{c h^{\prime}(x)}{h(x)+b / a} F(x) .
$$




\section{RECURRENCE RELATION OF QUOTIENT MOMENTS}

THEOREM 2.1. For $1 \leq r \leq s-2, k \geq 1, i=0,1,2, \ldots$ and $j=1,2, \ldots$,

$$
\begin{aligned}
& E\left[\frac{X^{* i}(r, n, m, k)}{X^{* j}(s, n, m, k)}\right]=\frac{j}{c \gamma_{s}} E\left[\frac{X^{* i}(r, n, m, k) h(X(s, n, m, k))}{X^{* j+1}(s, n, m, k) h^{\prime}(X(s, n, m, k))}\right] \\
& +\frac{j b}{a c \gamma_{s}} E\left[\frac{X^{* i}(r, n, m, k)}{X^{* j+1}(s, n, m, k) h^{\prime}(X(s, n, m, k))}\right]+E\left[\frac{X^{* i}(r, n, m, k)}{X^{* j}(s-1, n, m, k)}\right] .
\end{aligned}
$$

PROOF. We have from (1.3)

$$
\begin{aligned}
& E\left[\frac{X^{* i}(r, n, m, k)}{X^{* j+1}(s, n, m, k)}\right]=\frac{C_{s-1}}{(r-1) !(s-r-1) !} \int_{\alpha}^{\beta} \int_{\alpha}^{x} \frac{x^{i}}{y^{j+1}}[F(x)]^{m} f(x) g_{m}^{r-1}(F(x)) \\
& \quad \times\left[h_{m}(F(y))-h_{m}(F(x))\right]^{s-r-1}[F(y)]^{\gamma_{s}-1} f(y) d y d x .
\end{aligned}
$$

On using (1.7), we obtain

$$
\begin{aligned}
& E\left[\frac{X^{* i}(r, n, m, k)}{X^{* j+1}(s, n, m, k)} \Phi(X(s, n, m, k))\right] \\
& \quad=\frac{c C_{s-1}}{(r-1) !(s-r-1) !} \int_{\alpha}^{\beta} x^{i}[F(x)]^{m} f(x) g_{m}^{r-1}(F(x)) I(x) d x
\end{aligned}
$$

where

$$
\begin{aligned}
& \Phi(y)=[h(y)+b / a] / h^{\prime}(y) \text { and } \\
& I(x)=\int_{\alpha}^{x} \frac{1}{y^{j+1}}\left[h_{m}(F(y))-h_{m}(F(x))\right]^{s-r-1}[F(y)]^{\gamma_{s}} d y
\end{aligned}
$$

Integrating $I(x)$ by parts treating $\frac{1}{y^{j+1}}$ for integration and the rest of the integrand for differentiation, we get

$$
\begin{aligned}
I(x) & =\frac{\gamma_{s}}{j} \int_{\alpha}^{x} \frac{1}{y^{j}}\left[h_{m}(F(y))-h_{m}(F(x))\right]^{s-r-1}[F(y)]^{\gamma_{s}-1} f(y) d y \\
& -\frac{(s-r-1)}{j} \int_{\alpha}^{x} \frac{1}{y^{j}}\left[h_{m}(F(y))-h_{m}(F(x))\right]^{s-r-2}[F(y)]^{\gamma_{s}+m} f(y) d y .
\end{aligned}
$$


Substituting the value of $I(x)$ in (2.2) and simplifying the resulting expression we get the result given in (2.1).

REMARK 2.1. Putting $m=0, k=1$, in (2.1), we obtain a recurrence relation for quotient moment of order statistics as

$$
\begin{aligned}
& E\left(\frac{X_{n-r+1: n}^{i}}{X_{n-s+1: n}^{j}}\right)=\frac{j}{c(n-s+1)} E\left(\frac{X_{n-r+1: n}^{i} h\left(X_{n-s+1: n}\right)}{X_{n-s+1: n}^{j+1} h^{\prime}\left(X_{n-s+1: n}\right)}\right) \\
& \quad+\frac{j b}{a c(n-s-1)} E\left(\frac{X_{n-r+1: n}^{i}}{X_{n-s+1: n}^{j+1} h^{\prime}\left(X_{n-s+1: n}\right)}\right)+E\left(\frac{X_{n-r+1: n}^{i}}{X_{n-s+2: n}^{j}}\right) .
\end{aligned}
$$

REMARK 2.2. Setting $m=-1$ and $k \geq 1$ in theorem 2.1 , we get a recurrence relation for quotient moment lower $k$ record as

$$
\begin{gathered}
E\left(\frac{X_{L(r): k}^{i}}{X_{L(s): k}^{j}}\right)=E\left(\frac{X_{L(r): k}^{i}}{X_{L(s-1): k}^{j}}\right)+\frac{j}{c k} E\left(\frac{X_{L(r): k}^{i} h\left(X_{L(s): k}\right)}{X_{L(s): k}^{j+1} h^{\prime}\left(X_{L(s): k}\right)}\right) \\
+\frac{j b}{a c k} E\left(\frac{X_{L(r): k}^{i}}{X_{L(s): k}^{j+1} h^{\prime}\left(X_{L(s): k}\right)}\right) .
\end{gathered}
$$

THEOREM 2.2. For $1 \leq r \leq s-2, k \geq 1, i=0,1,2, \ldots$ and $j=1,2, \ldots$,

$$
\begin{aligned}
& E\left[\frac{X^{* i}(r, n, m, k) h(X(r, n, m, k))}{X^{* j}(s, n, m, k) h^{\prime}(X(r, n, m, k))}\right]+\frac{b}{a} E\left[\frac{X^{* i}(r, n, m, k)}{X^{* j}(s, n, m, k) h^{\prime}(X(r, n, m, k))}\right] \\
& \quad=\frac{c \gamma_{s}}{i+1} E\left[\frac{X^{* i+1}(r-1, n, m, k)}{X^{* j}(s-1, n, m, k)}\left(\frac{a h(X(r, n, m, k))+b}{a h(X(s-1, n, m, k))+b}\right)^{c(m+1)}\right] \\
& -\frac{c \gamma_{s}}{i+1} E\left[\frac{X^{* i+1}(r, n, m, k)}{X^{* j}(s-1, n, m, k)}\left(\frac{a h(X(r-1, n, m, k))+b}{\operatorname{ah}(X(s-1, n, m, k))+b}\right)^{c(m+1)}\right] \\
& -\frac{c(m+1)}{i+1} E\left[\frac{X^{* i+1}(r, n, m, k)}{X^{* j}(s, n, m, k)}\right] .
\end{aligned}
$$


PROOF. We have from (1.3)

$$
\begin{aligned}
& E\left[\frac{X^{* i}(r, n, m, k)}{X^{* j}(s, n, m, k)} \Phi(X(r, n, m, k))\right] \\
& \quad=\frac{c C_{s-1}}{(r-1) !(s-r-1) !} \int_{\alpha}^{\beta} \frac{1}{y^{j}}[F(y)]^{\gamma_{s}-1} f(y) I(y) d y,
\end{aligned}
$$

where

$$
I(y)=\int_{y}^{\beta} x^{i}[F(x)]^{m+1} g_{m}^{r-1}(F(x))\left[h_{m}(F(y))-h_{m}(F(x))\right]^{s-r-1} d x .
$$

Integrating $I(y)$ by parts treating $x^{i}$ for integration and the rest of the integrand for differentiation, we get

$$
\begin{aligned}
I(y) & =-\frac{(m+1)}{i+1} \int_{y}^{\beta} x^{i+1}[F(x)]^{m} f(x) g_{m}^{r-1}(F(x))\left[h_{m}(F(y))-h_{m}(F(x))\right]^{s-r-1} d x \\
& +\frac{(r-1)}{i+1} \int_{y}^{\beta} x^{i+1}[F(x)]^{2 m+1} f(x) g_{m}^{r-2}(F(x))\left[h_{m}(F(y))-h_{m}(F(x))\right]^{s-r-1} d x \\
& -\frac{(s-r-1)}{i+1} \int_{y}^{\beta} x^{i+1}[F(x)]^{2 m+1} f(x) g_{m}^{r-1}(F(x))\left[h_{m}(F(y))-h_{m}(F(x))\right]^{s-r-2} d x
\end{aligned}
$$

Substituting the value of $I(y)$ in (2.6) and simplifying the resulting expression we get the result given in (2.5).

REMARK 2.3. Putting $m=0, k=1$, in (2.5), we obtain a recurrence relation for quotient moment of order statistics as

$$
\begin{gathered}
E\left[\frac{X_{n-r+1: n}^{i} h\left(X_{n-r+1: n}\right)}{X_{n-s+1: n}^{j} h^{\prime}\left(X_{n-r+1: n}\right)}\right]+\frac{b}{a} E\left[\frac{X_{n-r+1: n}^{i}}{X_{n-s+1: n}^{j} h^{\prime}\left(X_{n-r+1: n}\right)}\right]=-\frac{c}{i+1}\left[\frac{E\left[X_{n-r+1: n}^{i+1}\right]}{E\left[X_{n-s+1: n}^{j}\right]}\right] \\
+\frac{c(n-s+1)}{i+1} E\left[\frac{X_{n-r+2: n}^{i+1}}{X_{n-s+2: n}^{j}}\left(\frac{a h\left(X_{n-r+2: n}\right)+b}{h^{\prime}\left(X_{n-s+2: n}\right)+b}\right)^{c}\right] \\
-\frac{c(n-s+1)}{i+1} E\left[\frac{X_{n-r+1: n}^{i+1}}{X_{n-s+2: n}^{j}}\left(\frac{a h\left(X_{n-r+1: n}\right)+b}{h^{\prime}\left(X_{n-s+2: n}\right)+b}\right)^{c}\right] .
\end{gathered}
$$


REMARK 2.4. Setting $m=-1$ and $k \geq 1$ in theorem 2.2, we get a recurrence relation for quotient moment lower $k$ record as

$$
\begin{gathered}
E\left(\frac{X_{L(r): k}^{i} h\left(X_{L(r): k}\right)}{X_{L(s): k}^{j} h^{\prime}\left(X_{L(r): k}\right)}\right)+\frac{b}{a} E\left(\frac{X_{L(r): k}^{i}}{X_{L(s): k}^{j} h^{\prime}\left(X_{L(r): k}\right)}\right) \\
=\frac{c k}{i+1}\left\{E\left(\frac{X_{L(r-1): k}^{i+1}}{X_{L(s-1): k}^{j}}\right)-E\left(\frac{X_{L(r): k}^{i+1}}{X_{L(s-1): k}^{j}}\right)\right\} .
\end{gathered}
$$

\section{RECURRENCE RELATION OF QUOTIENT CONDITIONAL EXPECTATION}

THEOREM 3.1. For the distribution as given in (1.6) and for $1 \leq r<s \leq n-2$, $i=0,1, \ldots, j=1,2 \ldots$ and $k=1,2, \ldots$,

$$
\begin{gathered}
E\left[\frac{X^{* i}(r, n, m, k)}{X^{* j}(s, n, m, k)} \mid X^{*}(r, n, m, k)=x\right]=E\left[\frac{X^{* i}(r, n, m, k)}{X^{* j}(s-1, n, m, k)} \mid X^{*}(r, n, m, k)=x\right] \\
\quad+\frac{j}{c \gamma_{s}} E\left[\frac{X^{* i}(r, n, m, k) h(X(s, n, m, k))}{X^{* j+1}(s, n, m, k) h^{\prime}(X(s, n, m, k))} \mid X^{*}(r, n, m, k)=x\right] \\
+\frac{j b}{a c \gamma_{s}} E\left[\frac{X^{* i}(r, n, m, k)}{X^{* j+1}(s, n, m, k) h^{\prime}(X(s, n, m, k))} \mid X^{*}(r, n, m, k)=x\right]
\end{gathered}
$$

and

$$
\begin{aligned}
& E\left[\frac{X^{* i}(r, n, m, k)}{X^{* j}(r+1, n, m, k)} \mid X^{*}(r, n, m, k)=x\right]=x^{i-j} \\
& \quad+\frac{j}{c \gamma_{r+1}} E\left[\frac{X^{* i}(r, n, m, k) h(X(r+1, n, m, k))}{X^{* j+1}(r+1, n, m, k) h^{\prime}(X(r+1, n, m, k))} \mid X^{*}(r, n, m, k)=x\right] \\
& \quad+\frac{j b}{a c \gamma_{r+1}} E\left[\frac{X^{* i}(r, n, m, k)}{X^{* j+1}(r+1, n, m, k) h^{\prime}(X(r+1, n, m, k))} \mid X^{*}(r, n, m, k)=x\right] .
\end{aligned}
$$


PROOF. From (1.4) and (1.7), we obtain

$$
\begin{gathered}
E\left[\frac{X^{* i}(r, n, m, k)}{X^{* j+1}(s, n, m, k)} \Phi\left(X^{*}(s, n, m, k)\right) \mid X^{*}(r, n, m, k)=x\right] \\
=\frac{c x^{i} C_{s-1}}{C_{r-1}(s-r-1) ![F(x)]^{\gamma_{r+1}}} I(x)
\end{gathered}
$$

where $I(x)$ is defined in (2.3). Substituting the value of $I(x)$ from (2.4) in (3.3) and simplifying the resulting expression we get the result given in (3.1).

When $s=r+1$

$$
I(x)=\int_{\alpha}^{x} \frac{1}{y^{j+1}}[F(y)]^{\gamma_{r+1}} d y .
$$

Integrating by parts and substituting the resulting expression in (3.3) for $s=r+1$, we get the result given in (3.2).

REMARK 3.1. Putting $m=0, k=1$, in (3.1) and (3.2), we obtain a recurrence relation for quotient conditional moment of order statistics as

$$
\begin{array}{r}
E\left(\frac{X_{n-r+1: n}^{i}}{X_{n-s+1: n}^{j}} \mid X_{n-r+1: n}=x\right)=E\left(\frac{X_{n-r+1: n}^{i}}{X_{n-s+2: n}^{j}} \mid X_{n-r+1: n}=x\right) \\
\quad+\frac{j}{c(n-s+1)} E\left(\frac{X_{n-r+1: n}^{i} h\left(X_{n-s+1: n}\right)}{X_{n-s+1: n}^{j+1} h^{\prime}\left(X_{n-s+1: n}\right)} \mid X_{n-r+1: n}=x\right) \\
+\frac{j b}{a c(n-s-1)} E\left(\frac{X_{n-r+1: n}^{i}}{X_{n-s+1: n}^{j+1} h^{\prime}\left(X_{n-s+1: n}\right)} \mid X_{n-r+1: n}=x\right)
\end{array}
$$

and

$$
\begin{aligned}
& E\left(\frac{X_{n-r+1: n}^{i}}{X_{n-r: n}^{j}} \mid X_{n-r+1: n}=x\right)=x^{i-j}+\frac{j}{c(n-r)} E\left(\frac{X_{n-r+1: n}^{i} h\left(X_{n-r: n}\right)}{X_{n-r: n}^{j+1} h^{\prime}\left(X_{n-r: n}\right)} \mid X_{n-r+1: n}=x\right) \\
& \quad+\frac{j b}{a c(n-r)} E\left(\frac{X_{n-r+1: n}^{i}}{X_{n-r: n}^{j+1} h^{\prime}\left(X_{n-r: n}\right)} \mid X_{n-r+1: n}=x\right) .
\end{aligned}
$$


REMARK 3.2 Setting $m=-1$ and $k \geq 1$ in theorem 2.1, we get a recurrence relation for quotient conditional moment lower $k$ record as

$$
\begin{aligned}
& E\left(\frac{X_{L(r): k}^{i}}{X_{L(s): k}^{j}} \mid X_{L(r): k}=x\right)=E\left(\frac{X_{L(r): k}^{i}}{X_{L(s-1): k}^{j}} \mid X_{L(r): k}=x\right) \\
& +\frac{j}{c k} E\left(\frac{X_{L(r): k}^{i} h\left(X_{L(s): k}\right)}{X_{L(s): k}^{j+1} h^{\prime}\left(X_{L(s): k}\right)} \mid X_{L(r): k}=x\right)+\frac{j b}{a c k} E\left(\frac{X_{L(r): k}^{i}}{X_{L(s): k}^{j+1} h^{\prime}\left(X_{L(s): k}\right)} \mid X_{L(r): k}=x\right)
\end{aligned}
$$

and

$$
\begin{aligned}
& E\left(\frac{X_{L(r): k}^{i}}{X_{L(r+1): k}^{j}} \mid X_{L(r): k}=x\right)=x^{i-j}+\frac{j}{c k} E\left(\frac{X_{L(r): k}^{i} h\left(X_{L(r+1): k}\right)}{X_{L(r+1): k}^{j+1} h^{\prime}\left(X_{L(r+1): k}\right)} \mid X_{L(r): k}=x\right) \\
& \quad+\frac{j b}{a c k} E\left(\frac{X_{L(r): k}^{i}}{X_{L(r+1): k}^{j+1} h^{\prime}\left(X_{L(r+1): k}\right)} \mid X_{L(r): k}=x\right) .
\end{aligned}
$$

THEOREM 3.2. For the distribution as given in (1.6) and for $1 \leq r<s \leq n-2$, $i=0,1, \ldots, j=1,2 \ldots$ and $k=1,2, \ldots$,

$$
\begin{aligned}
& E\left[\frac{X^{* i}(r, n, m, k) h(X(r, n, m, k))}{X^{* j}(s, n, m, k) h^{\prime}(X(r, n, m, k))} \mid X^{*}(s, n, m, k)=y\right] \\
& \quad+\frac{b}{a} E\left[\frac{X^{* i}(r, n, m, k)}{X^{* j}(s, n, m, k) h^{\prime}(X(r, n, m, k))} \mid X^{*}(s, n, m, k)=y\right] \\
& =\frac{c(s-1)}{(i+1) g_{m}(F(y))} E\left[\frac{X^{* i+1}(r-1, n, m, k)}{X^{* j}(s-1, n, m, k)} \mid X^{*}(s, n, m, k)=y\right] \\
& -\frac{c(s-1)}{(i+1) g_{m}(F(y))} E\left[\frac{X^{* i+1}(r, n, m, k)}{X^{* j}(s-1, n, m, k)} \mid X^{*}(s, n, m, k)=y\right]
\end{aligned}
$$




$$
\begin{aligned}
& +\frac{c(m+1) r}{i+1} E\left[\frac{X^{* i+1}(r+1, n, m, k)}{X^{* j}(s, n, m, k)} \mid X^{*}(s, n, m, k)=y\right] \\
& -\frac{c(m+1) r}{i+1} E\left[\frac{X^{* i+1}(r, n, m, k)}{X^{* j}(s, n, m, k)} \mid X^{*}(s, n, m, k)=y\right]
\end{aligned}
$$

and

$$
\begin{aligned}
& E\left[\frac{X^{* i}(r, n, m, k) h(X(r, n, m, k))}{X^{* j}(r+1, n, m, k) h^{\prime}(X(r, n, m, k))} \mid X^{*}(r+1, n, m, k)=y\right] \\
& \quad+\frac{b}{a} E\left[\frac{X^{* i}(r, n, m, k)}{X^{* j}(r+1, n, m, k) h^{\prime}(X(r, n, m, k))} \mid X^{*}(r+1, n, m, k)=y\right] \\
& =-\frac{r c}{(i+1) g_{m}(F(y))}\left\{y^{i-j+1}-E\left[\frac{X^{* i+1}(r-1, n, m, k)}{X^{* j}(r, n, m, k)} \mid X^{*}(r+1, n, m, k)=y\right]\right\} \\
& +\frac{c(m+1) r}{i+1}\left\{y^{i-j+1}-E\left[\frac{X^{* i+1}(r, n, m, k)}{X^{* j}(r+1, n, m, k)} \mid X^{*}(r+1, n, m, k)=y\right]\right\} . \quad(3.6)
\end{aligned}
$$

PROOF. From (1.5) and (1.7), we obtain

$$
\begin{gathered}
E\left[\frac{X^{* i}(r, n, m, k)}{X^{* j}(s, n, m, k)} \Phi\left(X^{*}(r, n, m, k)\right) \mid X^{*}(s, n, m, k)=y\right] \\
=\frac{c(s-1) !}{(r-1) !(s-r-1) ! y^{j} g_{m}^{s-1}(F(x))} I(y)
\end{gathered}
$$

where $I(y)$ is defined in (2.7). Substituting the value of $I(y)$ from (2.8) in (3.7) and simplifying the resulting expression we get the result given in (3.5).

When $s=r+1$

$$
I(y)=\int_{y}^{\beta} x^{i}[F(y)]^{m+1} g_{m}^{r-1}(F(x)) d x .
$$

Integrating by parts and substituting the resulting expression in (3.8) for $s=r+1$, we get the result given in (3.6). 
REMARK 3.3 Putting $m=0, k=1$, in (3.5) and (3.6), we obtain a recurrence relation for quotient conditional moment of order statistics as

$$
\begin{gathered}
E\left(\frac{X_{n-r+1: n}^{i} h\left(X_{n-r+1: n}\right)}{X_{n-s+1: n}^{j} h^{\prime}\left(X_{n-r+1: n}\right)} \mid X_{n-s+1: n}=y\right)+\frac{b}{a} E\left(\frac{X_{n-r+1: n}^{i}}{X_{n-s+1: n}^{j} h^{\prime}\left(X_{n-r+1: n}\right)} \mid X_{n-s+1: n}=y\right) \\
=\frac{c(s-1)}{(i+1)[1-F(x)]}\left\{E\left(\frac{X_{n-r+2: n}^{i+1}}{X_{n-s+2: n}^{j}} \mid X_{n-s+1: n}=y\right)-E\left(\frac{X_{n-r+1: n}^{i+1}}{X_{n-s+2: n}^{j}} \mid X_{n-s+1: n}=y\right)\right\} \\
\quad+\frac{c r}{(i+1)}\left\{E\left(\frac{X_{(n-r): n}^{i+1}}{X_{n-s+1: n}^{j}} \mid X_{n-s+1: n}=y\right)-E\left(\frac{X_{n-r+1: n}^{i+1}}{X_{n-s+1: n}^{j}} \mid X_{n-s+1: n}=y\right)\right\} .
\end{gathered}
$$

and

$$
\begin{aligned}
& E\left(\frac{X_{n-r+1: n}^{i} h\left(X_{n-r+1: n}\right)}{X_{n-r: n}^{j} h^{\prime}\left(X_{n-r+1: n}\right)} \mid X_{n-r+1: n}=y\right)+\frac{b}{a} E\left(\frac{X_{n-r+1: n}^{i}}{X_{n-r: n}^{j} h^{\prime}\left(X_{n-r+1: n}\right)} \mid X_{n-r: n}=y\right) \\
& =\frac{c r}{(i+1)[1-F(x)]}\left\{y^{i-j+1}-E\left(\frac{X_{n-r+2: n}^{i+1}}{X_{n-r+1: n}^{j}} \mid X_{n-r: n}=y\right)\right\} \\
& +\frac{c r}{(i+1)}\left\{y^{i-j+1}-E\left(\frac{X_{n-r+1: n}^{i+1}}{X_{n-r: n}^{j}} \mid X_{n-r: n}=y\right)\right\} .
\end{aligned}
$$

REMARK 3.4. Setting $m=-1$ and $k \geq 1$ in theorem 2.1 , we get a recurrence relation for quotient conditional moment lower $k$ record as

$$
\begin{aligned}
& E\left(\frac{X_{L(r): k}^{i} h\left(X_{L(r): k}\right)}{X_{L(s): k}^{j} h^{\prime}\left(X_{L(r): k}\right)} \mid X_{L(s): k}=y\right)+\frac{b}{a} E\left(\frac{X_{L(r): k}^{i}}{X_{L(s): k}^{j} h^{\prime}\left(X_{L(r): k}\right)} \mid X_{L(s): k}=y\right) \\
& =\frac{c(s-1)}{(i+1)[-\ln (F(y))]}\left\{E\left(\frac{X_{L(r-1): k}^{i+1}}{X_{L(s-1): k}^{j}} \mid X_{L(s): k}=x\right)-E\left(\frac{X_{L(r): k}^{i+1}}{X_{L(s-1): k}^{j}} \mid X_{L(s): k}=x\right)\right\}
\end{aligned}
$$

and 


$$
\begin{gathered}
E\left(\frac{X_{L(r): k}^{i} h\left(X_{L(r): k}\right)}{X_{L(r+1): k}^{j} h^{\prime}\left(X_{L(r): k}\right)} \mid X_{L(r+1): k}=y\right)+\frac{b}{a} E\left(\frac{X_{L(r): k}^{i}}{X_{L(r+1): k}^{j} h^{\prime}\left(X_{L(r): k}\right)} \mid X_{L(r+1): k}=y\right) \\
=\frac{c r}{(i+1)[-\ln (F(y))]}\left\{y^{i-j+1}-E\left(\frac{X_{L(r-1): k}^{i+1}}{X_{L(r): k}^{j}} \mid X_{L(r+1): k}=x\right)\right\}
\end{gathered}
$$

\begin{tabular}{|c|c|c|c|c|c|}
\hline Distribution & $F(x)$ & $a$ & $b$ & $c$ & $h(x)$ \\
\hline Inverse Weibull & $\begin{array}{c}e^{-(\theta / x)^{p}} \\
0<x<\infty\end{array}$ & 1 & 0 & $\theta^{p}$ & $e^{-x^{-p}}$ \\
\hline $\begin{array}{l}\text { Exponentiated } \\
\text { Weibull }\end{array}$ & $\begin{array}{l}{\left[1-e^{-(\lambda x)^{p}}\right]^{\tau}} \\
0<x<\infty\end{array}$ & 1 & 1 & $\tau$ & $-e^{-(\lambda x)^{p}}$ \\
\hline Power function & $\begin{array}{r}\quad(x / \lambda)^{p} \\
0<x<\lambda\end{array}$ & 1 & 0 & $p$ & $x / \lambda$ \\
\hline $\begin{array}{l}\text { Exponentiated } \\
\text { Pareto }\end{array}$ & $\begin{array}{c}{\left[1-(1+x)^{-\lambda}\right]^{\theta}} \\
0<x<\infty\end{array}$ & 1 & 1 & $\theta$ & $-(1+x)^{-\lambda}$ \\
\hline $\begin{array}{l}\text { Exponentiated } \\
\text { gamma }\end{array}$ & $\begin{array}{c}{\left[1-e^{-x}(x+1)\right]^{\theta}} \\
0<x<\infty\end{array}$ & 1 & 1 & $\theta$ & $-e^{-x}(x+1)$ \\
\hline $\begin{array}{l}\text { Generalized } \\
\text { exponential }\end{array}$ & $\begin{array}{l}{\left[1-e^{-\lambda x}\right]^{\theta}} \\
0<x<\infty\end{array}$ & 1 & 1 & $\theta$ & $-e^{-\lambda x}$ \\
\hline $\begin{array}{l}\text { Exponentiated } \\
\text { log-logistic }\end{array}$ & {$\left[\frac{(x / \sigma)^{\beta}}{1+(x / \sigma)^{\beta}}\right]^{\theta}$} & 1 & 0 & $\theta$ & $\frac{(x / \sigma)^{\beta}}{1+(x / \sigma)^{\beta}}$ \\
\hline
\end{tabular}

Table 3.1 Examples based on $d f \quad F(x)=[a h(x)+b]^{c}$. 


\begin{tabular}{|c|c|c|c|c|c|}
\hline & $0<x<\infty$ & & & & \\
\hline $\begin{array}{l}\text { Generalized } \\
\text { inverse Weibull }\end{array}$ & $\begin{array}{c}e^{-\theta(\alpha / x)^{\beta}} \\
0<x<\infty\end{array}$ & 1 & 0 & $\theta$ & $e^{-(\alpha / x)^{\beta}}$ \\
\hline $\begin{array}{l}\text { Extended type I } \\
\text { generalized } \\
\text { logistic }\end{array}$ & $\begin{array}{l}\left(\frac{\lambda}{\lambda+e^{-x}}\right)^{p} \\
-\infty<x<\infty\end{array}$ & 1 & 0 & $p$ & $\frac{\lambda}{\lambda+e^{-x}}$ \\
\hline Logistic & $\begin{array}{c}{\left[1+e^{-x}\right]^{-1}} \\
-\infty<x<\infty\end{array}$ & 1 & 1 & -1 & $e^{-x}$ \\
\hline Gumbel & $\begin{array}{c}\exp \left[-e^{-x}\right] \\
-\infty<x<\infty\end{array}$ & 1 & 0 & 1 & $e^{-e^{-x}}$ \\
\hline
\end{tabular}

Similarly several recurrence relations based on $d f F(x)=[a h(x)+b]^{c}$ can be established with proper choice of $a, b, c$ and $h(x)$.

\section{CHARACTERIZATION}

THEOREM 4.1. Let $X$ be a non-negative random variable having an absolutely continuous distribution function $F(x)$ with $F(0)=0$ and $0<F(x)<1$ for all $x>0$, then

$$
\begin{gathered}
E\left[\frac{X^{* i}(r, n, m, k)}{X^{* j}(s, n, m, k)} \mid X^{*}(r, n, m, k)=x\right]=E\left[\frac{X^{* i}(r, n, m, k)}{X^{* j}(s-1, n, m, k)} \mid X^{*}(r, n, m, k)=x\right] \\
\quad+\frac{j}{c \gamma_{s}} E\left[\frac{X^{* i}(r, n, m, k) h(X(s, n, m, k))}{X^{* j+1}(s, n, m, k) h^{\prime}(X(s, n, m, k))} \mid X^{*}(r, n, m, k)=x\right] \\
+\frac{j b}{a c \gamma_{s}} E\left[\frac{X^{* i}(r, n, m, k)}{X^{* j+1}(s, n, m, k) h^{\prime}(X(s, n, m, k))} \mid X^{*}(r, n, m, k)=x\right]
\end{gathered}
$$

if and only if 


$$
f(y)=\frac{c h^{\prime}(y)}{h(y)+b / a} F(y), \alpha \leq y \leq \beta .
$$

PROOF. The necessary part follows immediately from equation (3.1). On the other hand if the recurrence relation in equation (4.1) is satisfied, then on using equations (1.4), we have

$$
\begin{aligned}
& \frac{C_{s-1}}{C_{r-1}(s-r-1) !} \int_{\alpha}^{x} \frac{x^{i}}{y^{j}}\left[h_{m}(F(y))-h_{m}(F(x))\right]^{s-r-1} \frac{[F(y)]^{\gamma_{s}-1}}{[F(x)]^{\gamma_{r+1}}} f(y) d y \\
& =\frac{C_{s-2}}{C_{r-1}(s-r-2) !} \int_{\alpha}^{x} \frac{x^{i}}{y^{j}}\left[h_{m}(F(y))-h_{m}(F(x))\right]^{s-r-2} \frac{[F(y)]^{\gamma_{s-1}-1}}{[F(x)]^{\gamma_{r+1}}} f(y) d y \\
& +\frac{j C_{s-1}}{c \gamma_{s} C_{r-1}(s-r-1) !} \int_{\alpha}^{x} \frac{x^{i} h(y)}{y^{j+1} h^{\prime}(y)}\left[h_{m}(F(y))-h_{m}(F(x))\right]^{s-r-1} \frac{[F(y)]^{\gamma_{s}-1}}{[F(x)]^{\gamma_{r+1}}} f(y) d y \\
& +\frac{j b C_{s-1}}{a c \gamma_{s} C_{r-1}(s-r-1) !} \int_{\alpha}^{x} \frac{x^{i}}{y^{j+1} h^{\prime}(y)}\left[h_{m}(F(y))-h_{m}(F(x))\right]^{s-r-1} \frac{[F(y)]^{\gamma_{s}-1}}{[F(x)]^{\gamma_{r+1}}} f(y) d y
\end{aligned}
$$

Integrating the first integral on the right hand side of equation (4.2) by parts and simplifying the resulting expression, we get

$$
\begin{aligned}
& \frac{j C_{s-1}}{\gamma_{s} C_{r-1}(s-r-1) !} \int_{\alpha}^{x} \frac{x^{i}}{y^{j+1}}\left[h_{m}(F(y))-h_{m}(F(x))\right]^{s-r-1} \frac{[F(y)]^{\gamma_{s}-1}}{[F(x)]^{\gamma_{r+1}}} \\
& \quad \times\left\{-F(y)+\frac{h(y)}{c^{\prime}(y)} f(y)+\frac{b}{a c^{\prime}(y)} f(y)\right\} d y=0 .
\end{aligned}
$$

Now, applying a generalization of the Müntz-Szász Theorem (Hwang and Lin, [11]) to equation (4.3), we get

$$
f(y)=\frac{c h^{\prime}(y)}{h(y)+b / a} F(y), \quad \alpha \leq y \leq \beta .
$$




\section{CONCLUSION}

This paper deals with the lower generalized order statistics from the general class of distribution. Recurrence relations satisfied by the quotient moments and conditional quotient moments of lower generalized order statistics for a general class of distribution are derived. Characterization of this distribution by considering the recurrence relation of conditional expectation for general class of distribution satisfied by the quotient moment of the lower generalized order statistics. Special cases are also deduced.

\section{REFERENCES}

[1] Ahmad, A.A. (2001): Moments of order statistics from doubly truncated continuous distributions and characterizations, Statistics, Vol. 35, 479-494.

[2] Ahsanullah, M. (2004): A characterization of the uniform distribution by dual generalized order statistics, Communication of Statistics Theory and Methods, Vol. 33, 2921-2928.

[3] Asadi, M., Rao, C.R. and Shanbahag, D.N. (2001): Some unified characterization result on generalized Pareto distribution, Journal of Statistical Planning and Inference, Vol. 93, 29-50.

[4] Bieniek M and Szynal D (2003): Characterizations of distributions via linearity of regression of generalized order statistics, Metrika, Vol. 58, 259-271.

[5] Burkschat, M., Cramer, E. and Kamps, U. (2003): Dual generalized order statistics, Metron, Vol. LXI, 13-26.

[6] Chang, S.K. (2007): Recurrence relations of quotient moments of the Weibull distribution by record values. Journal of Applied Mathematics and Computing, Vol. 1, 471-477.

[7] Cramer, E. Kamps, U. and Keseling C. (2004): Characterization via linear regression of ordered random variables a unifying approach, Communication of Statistics Theory and Methods, Vol. 33, 2885-2911.

[8] El-Din, M.M.M. and Kotb, M.S. (2011): Recurrence relations for quotient moments of generalized order statistics and characterization, Journal of Advance Research in Statistics and Probability, Vol. 3, 1-14.

[9] Govindarajulu, Z. (2001): Characterization of double exponential using moments of order statistics, Communication of Statistics Theory and Methods, Vol. 30, 2355-2372.

[10] Grudzien, Z. and Szynal, D. (1998): On characterizations of continuous in terms of moments of order statistics when the sample size is random. Journal of Mathematical Sciences, Vol. 92, $4017-$ 4022.

[11] Hwang, J.S. and Lin, G.D. (1984): On a generalized moments problem II. Proc. American Mathematical Society, Vol. 91, 577-580.

[12] Kamps, U. (1995): A Concept of Generalized Order Statistics, B.G. Teubner Stuttgart, Germany.

[13] Kamps, U. (1998): Characterizations of distributions by recurrence relations and identities for moments of order statistics. In: Balakrishnan N and Rao CR, Handbook of Statistics 16, Order Statistics: Theory \& Methods, North-Holland, Amsterdam, Vol. 16, 291-311.

[14] Keseling, C. (1999): Conditional distributions of generalized order statistics and some characterizations, Metrika, Vol. 49, 27-40.

[15] Khan, A.H. and Abouammoh, A.M. (1999): Characterizations of distributions by conditional expectation of order statistics, Journal of Applied Statistical Sciences, Vol. 9, 159-168. 
[16] Khan, A.H., Khan, R.U. and Yaqub, M. (2006): Characterization of continuous distributions through conditional expectation of functions of generalized order statistics, Journal of Applied Probability and Statistics, Vol. 1, 115-131.

[17] Khan, R.U. and Kumar, D. (2010): On moments of lower generalized order statistics from exponentiated Pareto distribution and its characterization, Applied Mathematical Sciences (Ruse), Vol. 55, 2711-2722.

[18] Khan, R.U. and Kumar, D. (2011): Lower generalized order statistics from exponentiated gamma distribution and its characterization. ProbStats Forum, Vol. 4, 25-38.

[19] Khan, RU and Kumar, D. (2011): Expectation identities of lower generalized order statistics from generalized exponential distribution and a characterization. Mathematical Methods of Statistics, Vol. 20, 150-157.

[20] Kumar, D. (2011): Explicit expressions for moments of lower generalized order statistics from exponentiated Kumaraswamy distribution and its characterization. Journal of Applied Probability and Statistics, Vol. 6, 61-72.

[21] Kumar, D. (2012): Relations for moments of lower generalized order statistics from a family of Jshaped distributions and its characterization. Journal of Applied Probability and Statistics, Vol. 7, 71-86.

[22] Lee, M.Y. and Chang, S.K. (2004): Recurrence relations of quotient moments of the exponential distribution by record values. Honam Mathematical Journal, Vol. 26, 463-469.

[23] Lee, M.Y. and Chang, S.K. (2004): Recurrence relations of quotient moments of the Pareto distribution by record values. Pure and Applied Mathematics, Vol. 11, 97-102.

[24] Lee, M.Y. and Chang, S.K. (2004): Recurrence relations of quotient moments of the power function distribution by record values, Kangweon-Kyungki Mathematical Journal, vol. 12, 15-22.

[25] Mbah, A.K. and Ahsanullah, M. (2007): Some characterization of the power function distribution based on lower generalized order statistics, Pakistan Journal of Statistics, Vol. 23, 139-146.

[26] Pawlas, P. and Szynal, D. (2001): Recurrence relations for single and product moments of lower generalized order statistics from the inverse Weibull distribution, Demonstratio Mathematica, Vol. XXXIV, 353-358.

[27] Wu, J. and Ouyang, L.Y. (1996): On characterizing distributions by conditional expectations of functions of order statistics, Metrika, Vol. 34, 135-147.

\section{Devendra Kumar}

Department of Statistics, Amity Institute of Applied Sciences

Amity University, Noida-201303, India

Email:devendrastats@gmail.com 